\title{
Microbunch preserving bending system for a helical radiator at the European X-ray Free Electron Laser
}

\author{
Y. Li, ${ }^{1}$ W. Decking, ${ }^{2}$ B. Faatz, ${ }^{2}$ and J. Pflueger ${ }^{1}$ \\ ${ }^{1}$ European X-Ray Free Electron Laser (XFEL), Notkestrasse 85, 22603 Hamburg, Germany \\ ${ }^{2}$ Deutsches Elektronen Synchrotron (DESY), Notkestrasse 85, 22603 Hamburg, Germany
}

(Received 18 February 2010; published 10 August 2010)

\begin{abstract}
At the European X-ray Free Electron Laser there is a planar undulator system under construction called SASE3, which produces intense linearly polarized light in the wavelength range from 0.4-1.6 nm. Nevertheless there is a strong demand for circularly polarized radiation in this wavelength range. An important part of a potential solution is described in this paper. After the planar undulator the electron beam, which is completely bunched, is sent through a suitable radiator. This can be an economically and technically convenient method to generate radiation with polarization properties, which are determined only by the radiator. If in addition a bend is used to separate the light created by the linear SASE3 from that of the radiator, two beam lines may be served, one with planar and one with circular radiation. In this case the light of the helical radiator is not contaminated by the light generated by the planar system. In order to obtain coherent radiation in the radiator, the microbunching of the planar undulator must be preserved throughout the bend. This is the basic problem. In this paper a fundamental, basic study is made. Several solutions for bending systems are presented, whose complexities, wavelength ranges, and debunching effects are different. The expected circular polarization and radiation power by such a bend are simulated for a model radiator.
\end{abstract}

DOI: 10.1103/PhysRevSTAB.13.080705

PACS numbers: $41.60 . \mathrm{Cr}$

\section{INTRODUCTION}

The European X-ray Free Electron Laser (XFEL) [1] will generate $\mathrm{X}$ rays using the principle of self-amplified spontaneous emission (SASE) [2,3]. Currently there are three undulator lines foreseen: SASE1, SASE2, and SASE3. SASE1 and SASE2 are hard $x$-ray beam lines in the wavelength range of 0.1 to $0.4 \mathrm{~nm}$. SASE3 is a soft $\mathrm{x}$ ray undulator line which can generate radiation from 0.4 to $1.6 \mathrm{~nm}$ with a $17.5 \mathrm{GeV}$ electron beam by adjusting its undulator gap (see Table I). All undulators will be built as planar devices, which therefore supply linearly polarized radiation. There is, however, a strong demand in the user community for circularly polarized radiation at wavelengths exceeding $1 \mathrm{~nm}$. The study presented in this paper is an extension of the planned SASE3 baseline design and can be used to generate circularly polarized radiation.

Several possible helical schemes have been discussed in the context of SASE3. The first is replacing some of the last planar undulator segments by helical ones. The planar undulator prebunches the electron beam. The last 3-4 gain lengths are filled with helical undulator segments tuned to the same wavelength in order to generate powerful circularly polarized radiation. The drawback of this scheme is that a background of linearly polarized radiation cannot be avoided, which reduces the degree of circular polarization. A second proposal was made in Refs. [4,5]. In this scheme, a short orthogonal planar undulator is placed behind the long planar undulator. If the crossed undulator length is 1.3 times the FEL gain length, the two orthogonal linear components have equal intensities [6]. Therefore their combination results in circular polarization, if both intensities are equal and their phase difference is $90^{\circ}$. The FEL 3D gain length, however, depends on a number parameters such as wavelength, peak current, emittance, energy spread, $\beta$ function, etc. Some of them might fluctuate leading to fluctuations of the polarization as well.

TABLE I. Parameters of the European XFEL and the SASE3 undulator system.

\begin{tabular}{lcc}
\hline \hline Parameter & Value & Unit \\
\hline Electron beam energy & 17.5 & $\mathrm{GeV}$ \\
Normalized emittance, $\epsilon_{n}$ & 1.4 & $\mathrm{~mm} \mathrm{mrad}$ \\
Bunch charge & 1 & $\mathrm{nC}$ \\
Bunch peak current & 5 & $\mathrm{kA}$ \\
Energy spread after SASE3 & $>20.0$ & $\mathrm{MeV}$ \\
Undulator type & Planar & \\
Undulator period, $\lambda_{u}$ & 68 & $\mathrm{~mm}$ \\
rms undulator parameter, $K$ & $3.58-7.36$ & \\
Undulator segment length & 5 & $\mathrm{~m}$ \\
Intersection length & 1.1 & $\mathrm{~m}$ \\
Undulator cell length & 6.1 & $\mathrm{~m}$ \\
Number of cells & 21 & \\
SASE3 system length & 128.1 & $\mathrm{~m}$ \\
Radiation wavelength, $\lambda_{r}$ & $0.4-1.6$ & $\mathrm{~nm}$ \\
Average $\beta_{x, y}$ in undulator & 15 & $\mathrm{~m}$ \\
$\beta_{x, y}$ at undulator exit & $19 / 8$ & $\mathrm{~m}$ \\
$\alpha_{x, y}$ at undulator exit & 0.0 & \\
\hline \hline
\end{tabular}


In the option presented in this paper, the microbunching of the planar undulator is used. After the undulator, the electron beam is deflected by a bending system and subsequently passed through a helical radiator. If the microbunch structure of the buncher can be preserved, intense coherent radiation is emitted in the helical radiator. Already a quite short helical radiator can generate powerful circularly polarized radiation. In addition, linearly and circularly polarized radiation are well separated by the bending system. This option has two advantages: First, two independent beam lines can be served simultaneously, one with linear and the other with circular radiation. Moreover, the time structure in both beams is naturally synchronized. Second, the polarization properties of the radiator are completely independent from the light produced in the bunching undulator.

Using this scheme, the microbunching on the scale of the radiation wavelength, i.e. 0.4 to $1.6 \mathrm{~nm}$, which was formed in the planar undulator must be maintained throughout the bending system. This is a challenge especially considering the case of a large uncorrelated energy spread ( $>20 \mathrm{MeV}$ ) imprinted onto the electron beam by the FEL processes in the upstream SASE1 and SASE3 undulators [1].

Figure 1 illustrates the problem, which arises when a circularly symmetric macrobunch subdivided into microbunch slices is deflected by a single dipole. It is seen, that after passing the dipole, the microbunching is still preserved but only along its original direction. Figure 1 illustrates the first order geometric aberration of a uniform and circularly symmetric macrobunch subdivided into microbunches. It is bent by a single dipole. It is seen that, after passing the dipole, the microbunching is still preserved but only along its original direction. It needs to be rotated to its new direction by the bending angle $\alpha$.

Thus, the microbunching decreases with respect to the new orbit due to its rms length expansion. The expansion is determined by the product of the transverse beam size and the bending angle $\alpha$. As an example, suppose the trans-

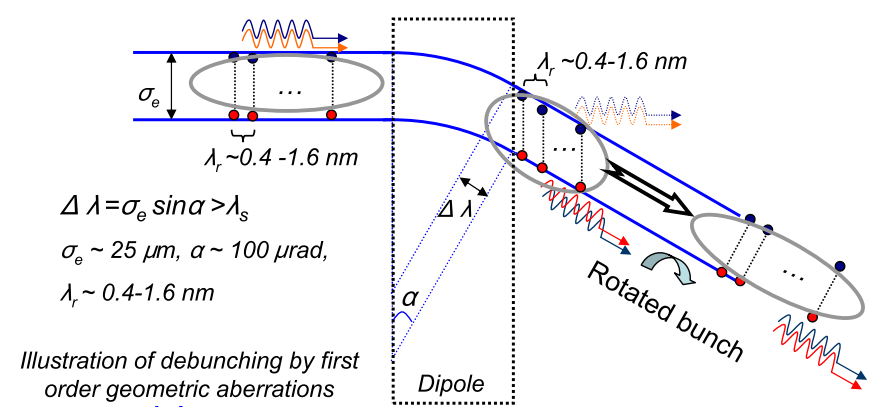

FIG. 1. (Color) Illustration of the bending of a circularly symmetric macrobunch subdivided into microbunch slices by a single dipole. The slices of the microbunches are indicated. The microbunching is only preserved along the original direction. verse size $\sigma_{e}=20 \mu \mathrm{m}$, the bending angle $\alpha$ is as small as $100 \mu \mathrm{rad}$, then the expansion is given by $\Delta L=\sigma_{e} \sin \alpha=$ $2 \mathrm{~nm}$. This is already more than the longest $1.6 \mathrm{~nm}$ wavelength in SASE3 with a $17.5 \mathrm{GeV}$ beam. Therefore, along the new orbit the microbunching is destroyed if no countermeasures are taken. In order to overcome this problem, the beam must be rotated as shown in Fig. 1. This can be done using one of the bend systems, which are described in this paper. Their complexities increase with increasing requirements on microbunch preservation.

In this paper not only the rms size of the microbunch length, which is of the order of nanometer, but also the fundamental bunch factor $b_{1}$ are used to describe the microbunch quality. $b_{1}$ is a parameter which normalizes microbunch length to the radiation wavelength. The higher the bunch factor, the better the microbunching. The bunch factor is defined as

$$
b_{1}=\left|\left\langle e^{-i \phi_{n}}\right\rangle\right|,
$$

where $\phi_{n}$ is the $n$th electron's ponderomotive phase, \langle\rangle means averaging over all electrons of the bunch.

All simulations presented here are based on the SASE3 parameters of the European XFEL as given in Table I.

The purpose of the bending system is to separate the linearly polarized from the circularly polarized radiation. The minimum bending angle required for an effective separation depends on the angular distribution of the photon beam. The divergences of both beams are assumed Gaussian and are calculated using 3D FEL codes. For SASE3 and $\lambda_{r}=1.6 \mathrm{~nm}$ the rms divergence is $11 \mu \mathrm{rad}$. Assuming a safe $9 \sigma$ separation results in a minimum bending angle of about $\alpha=100 \mu \mathrm{rad}$. This is considered to be a safe lower limit for the bending angle.

The beam dynamics analysis of this paper follows the notation used in Refs. [7,8]. The vector $X=\left(x, x^{\prime}, y, y^{\prime}\right.$, $\ell, \delta$ ) describes an electron in the six-dimensional phase space with respect to a virtually ideal electron. For such a reference electron all elements are zero by definition. The horizontal and vertical transverse coordinates are $(x, y)$, primed quantities are derivatives $\frac{d}{d s}$, where $s$ is the coordinate along the curved reference orbit. $\ell$ is the longitudinal position and $\delta$ is the fractional momentum deviation.

Because the preservation of microbunching is the main objective, we focus on the studies for the variation of $\ell$, which is denoted by $\Delta \ell$. Up to second order $\Delta \ell$ can be expressed as

$$
\Delta \ell=\sum_{i=1}^{6} R_{5 i} X_{i}+\sum_{i, j=1}^{6} T_{5 i j} X_{i} X_{j} .
$$

The $R_{5 i}$ and $T_{5 i j}$ are the first and second order transport elements, relevant for the longitudinal position. The rms bunch length is calculated as

$$
\sigma=\sqrt{\left\langle\Delta \ell_{n}^{2}\right\rangle-\left\langle\Delta \ell_{n}\right\rangle^{2}}
$$


where the subscript $n$ refers to the $n$th electron in the bunch. Note that $\Delta \ell<\lambda_{r}$.

This paper is organized as follows: After this introduction Sec. II studies different first order achromatic and isochronous bending systems, which only use dipoles and quadrupoles. The finite energy spread of the electron beam is taken into account by choosing different $R_{56}$ values appropriately.

Section III studies second order bending systems, where second order terms $T_{5 i j}$ are compensated using a repetitive cell structure including sextupoles. This method requires many magnetic components. Its potential is demonstrated for a $0.1 \mathrm{~nm}$ microbunch transported through the system. Based on these results a simplified system is studied, which can be used in the wavelength range of SASE3.

In Sec. IV the results are exemplified quantitatively. Microbunch distributions after the SASE3 undulator system are obtained using GENESIS 1.3. They are properly transported through the different bending sections as studied in Secs. II and III. The radiation properties in a test radiator are calculated and compared.

Finally Sec. V gives a summary and outlook.

\section{STUDY OF FIRST ORDER BENDING SYSTEMS}

\section{A. Analysis for bunch expansion by second order aberrations}

In first order bending systems using only dipoles and quadrupoles, second order aberrations cannot be avoided. Nevertheless their impact can be evaluated and, as will be demonstrated below, their contribution can be minimized. For the simulations, which are described in the following sections, the longitudinal electron distribution is described by a Gaussian. Its rms length, $\sigma$, is chosen such that $6 \sigma$ corresponds to the radiation wavelength, $\lambda_{r}$, which is in the range 0.4 to $1.6 \mathrm{~nm}$. Thus, more than $99 \%$ of the particles of a microbunch are tracked.

In order to estimate debunching effects not only the influence of first order term $R_{56}$ but also the second order terms must be taken into account. Second order geometric aberrations are described by the terms $T_{5 i j}$ for $i, j \neq 6$. They are distinguished from energy correlated aberrations with $i, j=6$. We use $\sigma_{\ell, g}$ to denote the bunch length expansion by second order geometric aberrations:

$$
\begin{aligned}
\sigma_{\ell, g}^{2}= & \epsilon^{2}\left[2\left(T_{511} \beta_{x 0}-T_{521} \alpha_{x 0}+T_{522} \gamma_{x 0}\right)^{2}+2\left(T_{533} \beta_{y 0}\right.\right. \\
& \left.-T_{543} \alpha_{y 0}+T_{544} \gamma_{y 0}\right)^{2}+\left(T_{521}^{2}-4 T_{511} T_{522}\right) \\
& \left.+\left(T_{543}^{2}-4 T_{533} T_{544}\right)\right],
\end{aligned}
$$

where $\epsilon$ denotes the geometrical emittance and $\alpha, \beta, \gamma$ are the Twiss parameters. $\sigma_{\ell, g}^{2}$ has its minimum value if the initial $\alpha_{0}$ and $\beta_{0}$ satisfy the following relations:

$$
\begin{aligned}
& \beta_{x 0}=\frac{2 T_{522}}{\sqrt{4 T_{511} T_{522}-T_{521}^{2}}}, \\
& \alpha_{x 0}=\frac{T_{521}}{\sqrt{4 T_{511} T_{522}-T_{521}^{2}}}, \\
& \beta_{y 0}=\frac{2 T_{544}}{\sqrt{4 T_{533} T_{544}-T_{543}^{2}}}, \\
& \alpha_{y 0}=\frac{T_{543}}{\sqrt{4 T_{533} T_{544}-T_{543}^{2}}},
\end{aligned}
$$

in this case, $\sigma_{\ell, g}^{2}$ reduces to

$$
\sigma_{\ell, g}^{2}=\epsilon^{2}\left[\left(4 T_{511} T_{522}-T_{521}^{2}\right)+\left(4 T_{533} T_{544}-T_{543}^{2}\right)\right] .
$$

The second order geometrical terms $T_{5 i j}$ at the end of the transport system can be deduced from $[7,8]$. We use their notation:

$$
\begin{aligned}
T_{511} & =\int_{0}^{L}\left[h\left(x \mid x_{0}^{2}\right)+\frac{1}{2} C_{x}^{\prime 2}\right] d s \\
T_{521} & =\int_{0}^{L}\left[h\left(x \mid x_{0} x_{0}^{\prime}\right)+C_{x}^{\prime} S_{x}^{\prime}\right] d s \\
T_{522} & =\int_{0}^{L}\left[h\left(x \mid x_{0}^{\prime 2}\right)+\frac{1}{2} S_{x}^{\prime 2}\right] d s \\
T_{533} & =\int_{0}^{L}\left[h\left(x \mid y_{0}^{2}\right)+\frac{1}{2} C_{y}^{\prime 2}\right] d s \\
T_{543} & =\int_{0}^{L}\left[h\left(x \mid y_{0} y_{0}^{\prime}\right)+C_{y}^{\prime} S_{y}^{\prime}\right] d s \\
T_{544} & =\int_{0}^{L}\left[h\left(x \mid y_{0}^{\prime 2}\right)+\frac{1}{2} S_{y}^{\prime 2}\right] d s .
\end{aligned}
$$

$L$ is the integration length of the whole system. $C_{x, y}, S_{x, y}$ are cosine- and sinus-like solutions of the equations of motion. $h$ denotes to the local curvature. The terms such as $\left(x \mid x_{0}^{2}\right)$ are the notations for the second order terms $T_{1 i j}$ at positions inside the system. They are functions of first order terms $C_{x, y}$ and $S_{x, y}$ whose expressions can be found in Refs. [7,8]. From Eq. (7) the second order terms of a magnet system, $T_{5 i j}$, can be calculated, then substituted into Eq. (6) and $\sigma_{\ell, g}$ can be calculated. In our case the dipoles are quite weak, so the second order expansion $\sigma_{\ell, g}$ is mostly induced by quadrupoles.

Usually, the Courant-Snyder parameters do not satisfy Eq. (5). Therefore a section is needed to match the $\alpha$ and $\beta$ to those minimizing the deterioration in the bend. The quadrupoles of the matching section might induce debunching, which should also be taken into account. Figure 2 shows some simulations for the matching section for parameters needed for SASE3. In this figure, four quadrupoles, shown at the top of the figure, are used to match the four parameters $\alpha_{x}, \alpha_{y}, \beta_{x}$, and $\beta_{y}$ to typical numbers based on Eq. (5). This simulation is done for 


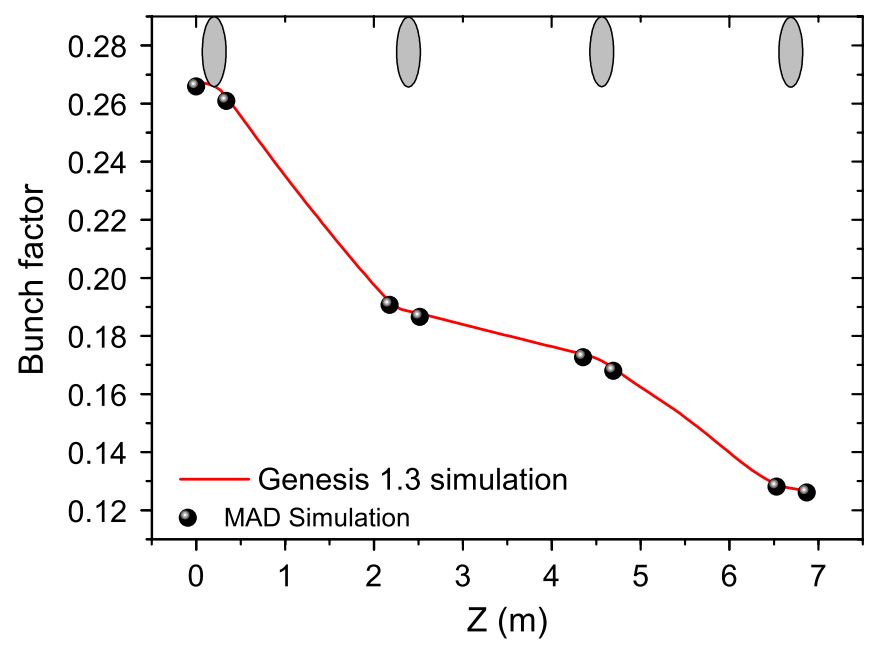

FIG. 2. (Color) Bunch factor development in matching section for $\alpha$ and $\beta$ according to Eq. (5). The code GENESIS 1.3 (solid line) and MAD8 (circles) are used for this simulation.

$0.4 \mathrm{~nm}$ microbunches. The codes GENESIS 1.3 [9] and MAD8 [10] are used and compared in Fig. 2. The line shows the results by GENESIS 1.3 and the dots show the result by MAD8. As can be seen, they match well. It is seen that in this example the bunch factor drops from 0.27 to 0.12 . Such a matching section always needs to be integrated into the first order bending systems, which are presented below.

\section{B. First order achromatic system}

In an achromatic bending system the final transverse position does not depend on energy deviation. A simple first order achromatic system can be comprised by two dipoles and one quadrupole in between. The whole system is mirror symmetric and in order to be achromatic to first order the quadrupole has to be adjusted such that off energy particles have no slope in the center of the quadrupole. It can be shown that in such a simple bending system the debunching is significant, especially if the matching section is included.

An improvement is achieved by adding two reverse dipoles adjacent to the quadrupole, as shown on the right side of Fig. 3. The angles of the two inner dipoles are

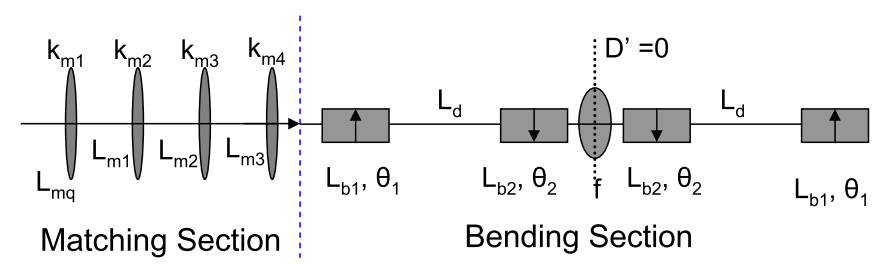

FIG. 3. (Color) First order achromatic bend comprised of four dipoles and one quadrupole. The two inner dipoles have opposite sign as compared to the two outer ones. Since $\left|\theta_{2}\right|<\left|\theta_{1}\right|$, the beam is deflected by the whole system. The matching section shown to the left is used to match $\alpha$ and $\beta$ according to Eq. (5). smaller than the two outer dipoles $\left(\left|\theta_{2}\right|<\left|\theta_{1}\right|\right)$, so the beam is still deflected by the whole system. With this configuration, the minimum geometric aberrations $\sigma_{\ell, g}^{2}$ can be calculated:

$$
\sigma_{\ell, g}^{2}=8 \epsilon^{2} \frac{\left(\left|\theta_{1}\right|-\left|\theta_{2}\right|\right)^{2}}{\theta_{1}^{2}} .
$$

If the total deflecting angle $2\left(\theta_{1}-\theta_{2}\right)$ is fixed, a larger $\theta_{1}$ means a smaller second order expansion $\sigma_{\ell, g}$. However, this system has a larger first order term $R_{56}$. Its contribution to microbunch expansion is

$$
\sigma_{R 56}^{2}=R_{56}^{2} \sigma_{\Delta E / E}^{2}=4\left(\frac{L_{b 1}+L_{b 2}}{2}+L_{d}\right)^{2} \theta_{1}^{2} \theta_{2}^{2} \sigma_{\Delta E / E}^{2} .
$$

$L_{b 1}, L_{b 2}$, and $L_{d}$ are explained in Fig. 3. $\sigma_{\Delta E / E}$ is the relative energy spread of the beam. In contrast to the case of $\sigma_{\ell, g}$, Eq. (8), a large angle $\theta_{1}$ means a larger $\sigma_{R 56}$. Therefore a careful balance between $\sigma_{\ell, g}$ and $\sigma_{R 56}$ is needed.

The following example estimates the properties of this system: Let $L_{b 1}=L_{b 2}=0.2 \mathrm{~m}, \quad L_{d}=1 \mathrm{~m}, \theta_{1}=$ $160 \mu \mathrm{rad}, \theta_{2}=-100 \mu \mathrm{rad}$, and $\sigma_{\Delta E / E}=1.14 \times 10^{-3}$, then it results in $\sigma_{\ell, g}=0.04 \mathrm{~nm}$ and $\sigma_{R 56}=0.04 \mathrm{~nm}$. Therefore the total bunch expansion is $\sqrt{\sigma_{\ell, g}^{2}+\sigma_{R 56}^{2}}=$ $0.06 \mathrm{~nm}$.

This evaluation requires matched $\alpha$ and $\beta$. The influence of the matching section should also be taken into account for parameter optimization.

To illustrate the influence by the whole first order achromatic bend, including the matching section, simulations using the code ELEGANT [11] have been done. Figure 4 shows the results. The initial bunch is assumed to be Gaussian. 20000 sample electrons are tracked through the bend and their positions in longitudinal phase space $(\ell, \delta)$ are shown as the dots in the plots.

The red histogram shows the statistic distribution of the electrons. All plots have the same scale: The horizontal scale shows the longitudinal coordinate. The zero is given by a reference particle with no deviation. The vertical scale to the left shows the particle density in counts. The right one shows the energy deviation $\Delta \gamma / \gamma$ of the particles. The two plots on the left represent the initial conditions of the microbunch before the bend at 0.4 (top) and $1.6 \mathrm{~nm}$ (bottom). The two plots on the right show the bunch properties after the bend. The longitudinal distributions were chosen according to $\sigma_{\ell}=\lambda_{r} / 6$. It is seen that the bunch factor $b_{1}$ for $0.4 \mathrm{~nm}$ drops from initially 0.573 to 0.007 and its rms length expands from 0.067 to $0.227 \mathrm{~nm}$. So at this wavelength there is complete debunching and the system is of no use. For $1.6 \mathrm{~nm}$, however, $b_{1}$ drops from initially 0.573 to only 0.399 , a loss of about $30 \%$, and its rms length expands from 0.267 to $0.343 \mathrm{~nm}$. So for $1.6 \mathrm{~nm}$ this system is of good use. 


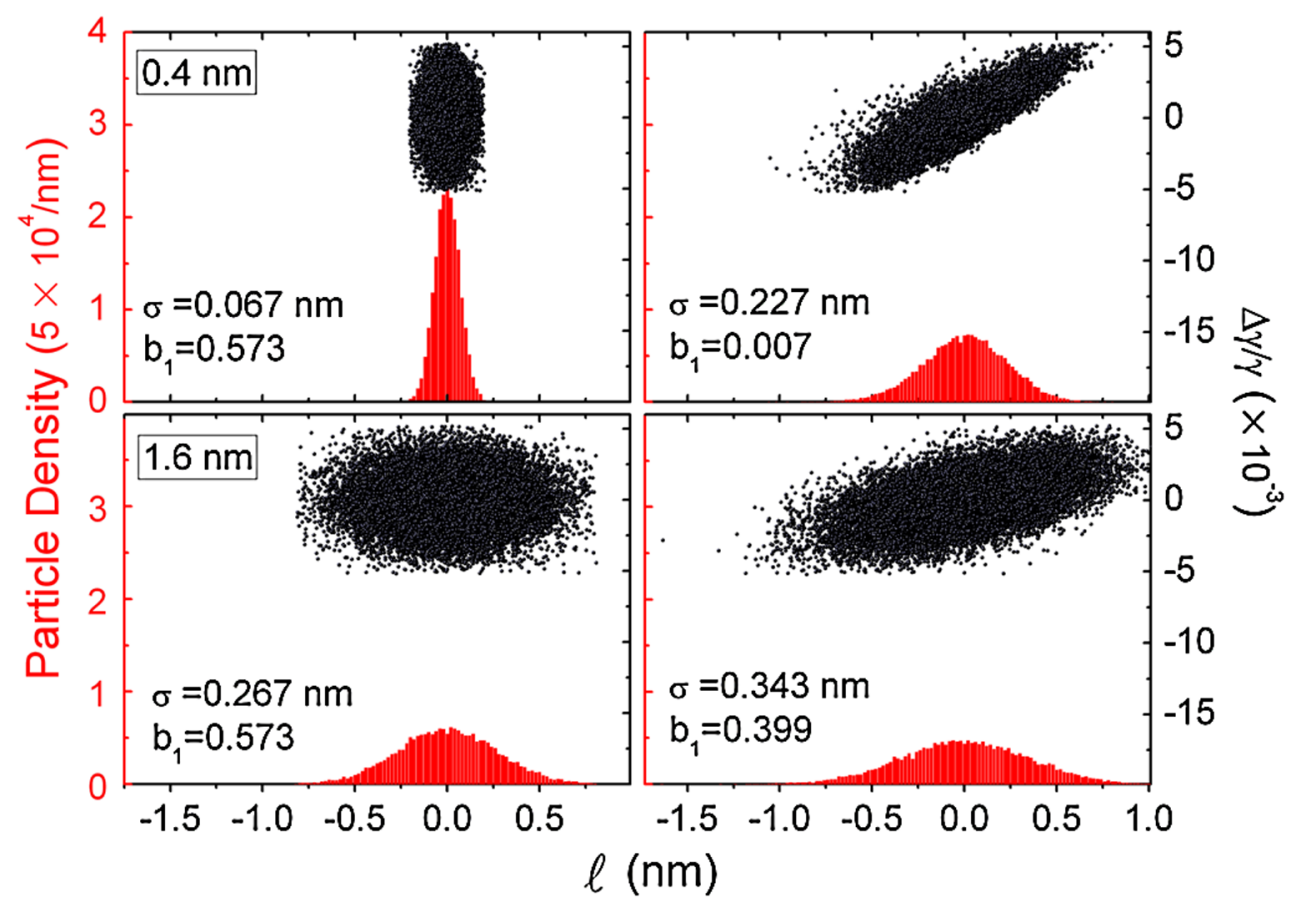

FIG. 4. (Color) Debunching properties of a first order achromatic bending system. Gaussian distributions for the longitudinal phase space are assumed. The top figures refer to $\lambda_{r}=0.4 \mathrm{~nm}$ and the bottom figures to $\lambda_{r}=1.6 \mathrm{~nm}$. $6 \sigma$ of the indicated microbunch lengths refers to the indicated $\lambda_{r}$.

Beam optical functions for the first order achromatic bend are show in Fig. 5.

\section{First order isochronous system}

In an isochronous bending system all electrons have the same path length. A simple system, which is isochronous to first order, can be comprised by three dipoles and two quadrupoles arranged in a mirror symmetric fashion. Figure 6 shows this configuration including the matching section. The central dipole magnet has an opposite sign as compared the two outer ones.

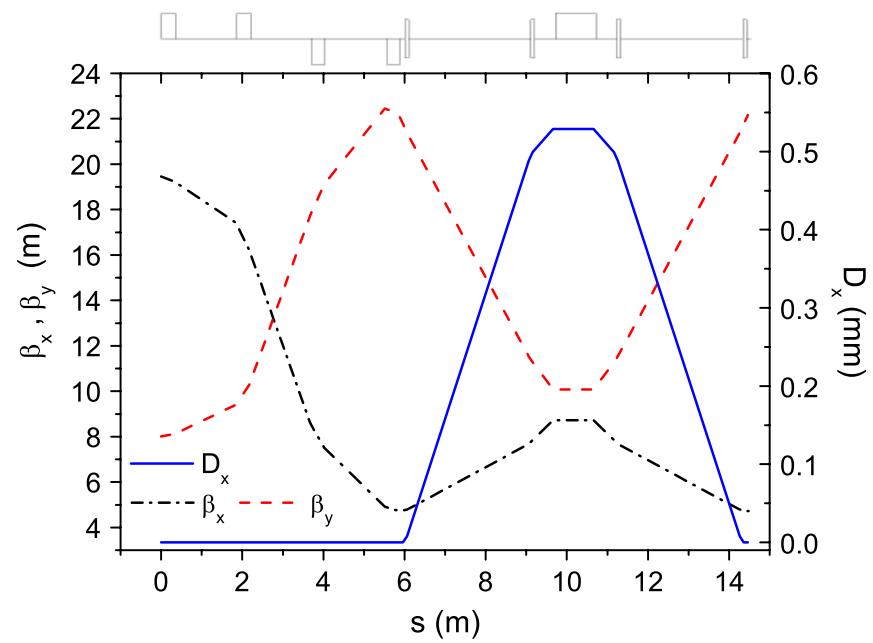

FIG. 5. (Color) Beam optical functions for the first order achromatic bend.
All first order terms $R_{5 i}$ are zero. Hence, the debunching is due to second order aberrations. If the dipoles are weak, they can be treated as drift spaces to calculate the second order terms $T_{5 i j}$ for this system. Using Eq. (6), the increase of the minimum $\sigma_{\ell, g}^{2}$ caused by this first order isochronous bend is given by

$$
\sigma_{\ell, g}^{2}=\frac{8 \epsilon^{2}}{f^{2}}\left[\left(\frac{l_{1} l_{2}}{f}\right)^{2}+l_{1}\left(l_{1}+l_{2}\right)\right]
$$

where $l_{1}=L_{b 1}+L_{d 1}$ and $l_{2}=L_{b 2}+L_{d 2}$ as shown in Fig. 6. $f$ is the focus length of the quadrupole in thin lens approximation. The parameters of $l_{1}, l_{2}$, and $f$ in Eq. (10) have to match the conditions for the first order isochronous bend. Examples for $l_{1}$ and $l_{2}$ are $\approx 1-3 \mathrm{~m}$ and for $f \approx$ $1 \mathrm{~m}$. This simple estimate shows that 2 nd order terms become significant.

The same analysis as shown in Fig. 4 has been made for the isochronous bending system. The results are shown in

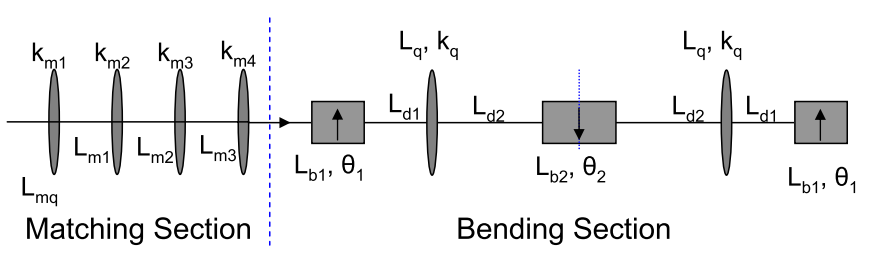

FIG. 6. Simple first order isochronous bend comprised of three dipoles and two quadrupoles. The inner dipole has an opposite sign as compared to the two outer ones. The matching section used to match $\alpha$ and $\beta$ according to Eq. (5) is shown in the left. 


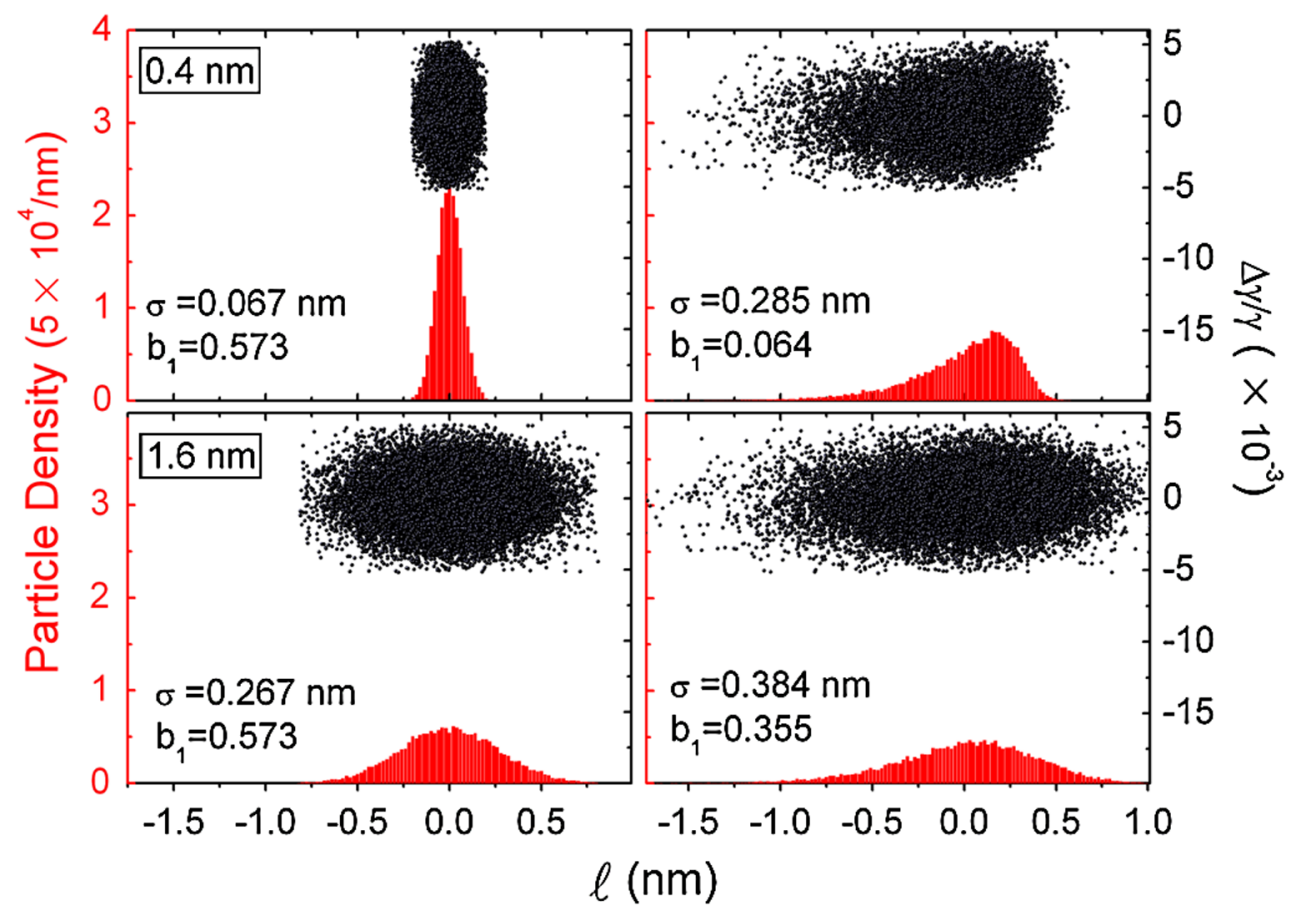

FIG. 7. (Color) Debunching properties of a first order isochronous bending system for $\lambda_{r}=0.4 \mathrm{~nm}$ (top) and $\lambda_{r}=1.6 \mathrm{~nm}$ (bottom). The treatment is identical to that used in Fig. 4.

Fig. 7. It is seen that, similar to the first order achromatic system, the $0.4 \mathrm{~nm}$ microbunch is seriously expanded. For the longer $1.6 \mathrm{~nm}$ bunch, the debunching is more moderate, but larger than the achromatic case. This illustrates the influence of the second order terms.

Beam optical functions for the first order isochronous bend are shown in Fig. 8.

\section{Debunching in an undulator behind the bend}

Both first order bending systems presented in the previous sections have similar properties with respect to mi-

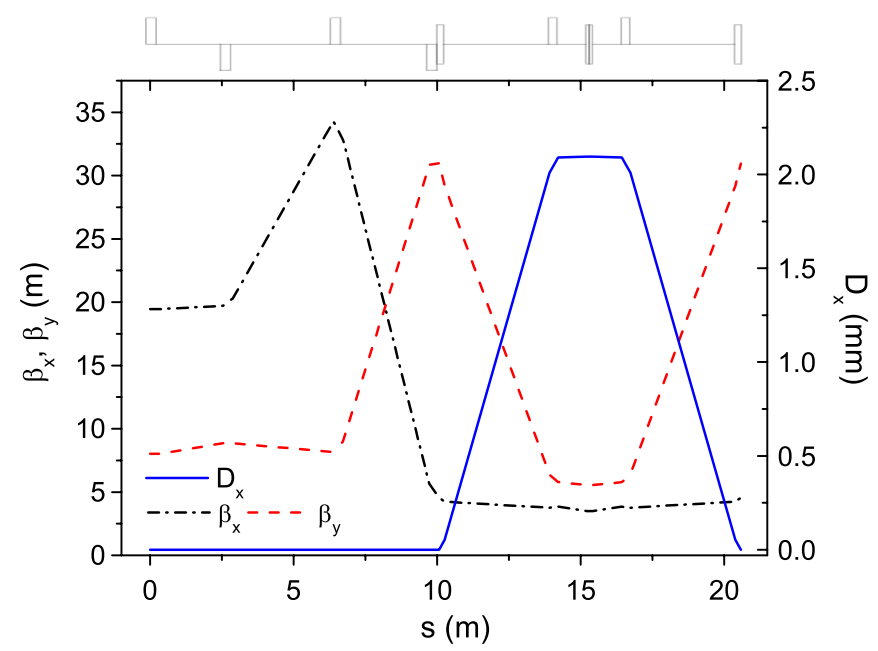

FIG. 8. (Color) Beam optical functions for the first order isochronous bend. crobunching. Both of them can only be used for radiation wavelengths longer than $\approx 1 \mathrm{~nm}$. However, by careful comparison of Figs. 4 and 7 a difference can be observed: For the achromatic bend particles are rotated clockwise in the $(\ell, \delta)$ phase space. But for the isochronous bend this is not the case. In the case of the first order achromat a nonzero $R_{56}$ plays the dominant role for the rotation. The first order isochronous bend compensates all first order terms $R_{5 i}$ and therefore particles are not rotated in the $(\ell, \delta)$ phase space. The impact of the $R_{56}$ to bunch expansion is of special importance for the undulator, which will be used after the bending system. The longitudinal position of the $n$th electron after the bend is $\Delta \ell_{n}=-R_{56} \delta_{n}$. Its ponderomotive phase is

$$
\phi_{n}=\phi_{0 n}-\frac{R_{56} \delta_{n}}{\lambda_{r}} \pi
$$

where $\phi_{0 n}$ is its initial phase before bend. The development of $\phi_{n}$ in the undulator is given by

$$
\frac{d \phi_{n}}{d \tau}=2 \delta_{n}, \quad \frac{d \delta_{n}}{d \tau}=-2 \frac{k_{r} a_{r} a_{u}}{k_{u} \gamma}
$$

Here $\tau=k_{u} z, k_{u}=\frac{2 \pi}{\lambda_{u}}$, and $\lambda_{u}$ is the undulator period. $z$ is the coordinate along the undulator axis. $\tau=0$ corresponds to the position at the beginning of the undulator. Note that $\tau \geq 0 . k_{r}=\frac{2 \pi}{\lambda_{r}}$, with $\lambda_{r}$ the radiation wavelength. $a_{u}$ is the vector potential of the undulator field and $a_{r}$ that of the laser field. Since after the bend the new radiation field just 
builds up $a_{r}$ is negligibly small. Therefore $\delta_{n}$ is constant over the radiator. The phase of the $n$th electron inside the undulator can be described by

$$
\phi_{n}(\tau)=\phi_{0 n}+2 \delta_{n}\left(\tau-\frac{R_{56} \pi}{\lambda_{r}}\right)
$$

The evolution of the bunching over the undulator is calculated by substitution of Eq. (13) into Eq. (1):

$$
\frac{b_{1}(\tau)}{b_{1, i}}=e^{-2\left[\tau-\left(R_{56} \pi / \lambda_{r}\right)\right]^{2} \sigma_{\Delta E / E}^{2}} \approx 1-2\left(\tau-\frac{R_{56} \pi}{\lambda_{r}}\right)^{2} \sigma_{\Delta E / E}^{2}
$$

Here $b_{1, i}$ is the bunch factor before the bend. $b_{1}(\tau)$ is the bunch factor inside the undulator at position $\tau$. Thus, Eq. (14) indicates that there is microbunch evolution not only in the bending system, but also in the undulator after the bend. From this equation it is seen that the sign of $R_{56}$ is important.

For illustration of Eq. (14), Fig. 9 shows the bunch factor evolution in a $6 \mathrm{~m}$ long undulator with three different values for $R_{56}$. These results were obtained using GENESIS 1.3.

In Fig. 9, the black square line represents the case of $R_{56}=0$ : the bunch factor drops parabolically. The red dotted line shows the case of $R_{56}=-35 \mathrm{~nm}$ : Initially it is lower than the black square line and it drops faster as well. The blue triangle line shows the case of $R_{56}=$

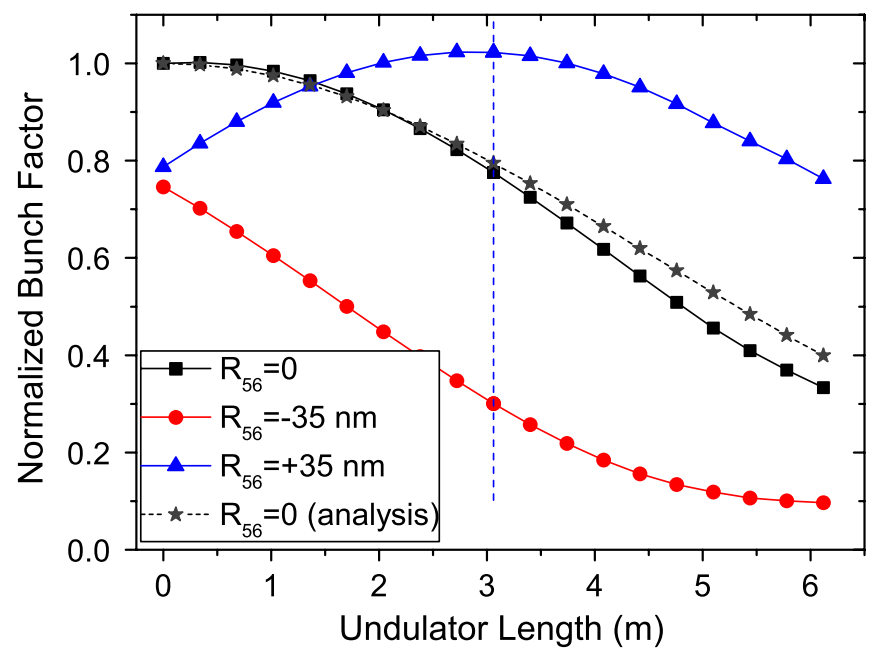

FIG. 9. (Color) Evolution of the bunch factor in an undulator placed after the bending section. Its length is $6 \mathrm{~m}$, the other parameters are given in Table I. Three cases are shown: The black square line denotes $R_{56}=0$, the red dotted line denotes $R_{56}=-35 \mathrm{~nm}$, and the blue triangle line denotes $R_{56}=$ $+35 \mathrm{~nm}$. These results were calculated using GENESIS 1.3. Analytic results using Eq. (14) for $R_{56}=0$ are shown by the star line.
$+35 \mathrm{~nm}$ : at the begin of the undulator, the bunch factor is as small as the case of a negative $R_{56}$, but it parabolically increases first. In the center of the undulator at $3 \mathrm{~m}$, the bunch factor has its maximum, then it drops to its initial value. On average it has the highest value. For comparison the green star line shows analytic results for $R_{56}=0$ using Eq. (14). In the first half of the undulator it matches the black square line quite well. The deviation in the second half shows that the assumption leading to Eq. (12) is no longer valid: With increasing undulator length the radiation field builds up so that $a_{r}$ may not be neglected.

From this analysis it is obvious that a positive $R_{56}$ in the bending system has the most benefits. The two examples shown above have either negative or zero $R_{56}$. For an achromatic bend as shown in Fig. 3 it is impossible to achieve a positive or even zero $R_{56}$ by tuning the magnet strengths. An isochronous bend as shown in Fig. 6, however, already has a zero $R_{56}$. Furthermore it is possible to detune the dipole magnets in such a way as to obtain a positive $R_{56}$. In this case this system is not isochronous anymore. It is achromatic with a positive $R_{56}$. As shown above these properties favor the use of a first order isochronous bend.

\section{SECOND ORDER BENDING SYSTEMS INCLUDING SEXTUPOLES}

In the previous sections it has been demonstrated that first order achromatic or isochronous bending systems work for wavelengths above $1 \mathrm{~nm}$, but due to nonzero second order terms $T_{5 i j}$ they cannot be used at $0.4 \mathrm{~nm}$. A straightforward solution is to compensate all second order terms $T_{5 i j}$ and set the first order terms $R_{5 i}$ to zero. Additionally, in order to avoid the matching section the transport matrix of the whole bending system should be unity.

\section{A. Compensation of the $T_{5 i j}$ elements by a structure of repetitive cells}

In Refs. [7,8] Brown et al. gives a solution for a second order achromat. They set $T_{i j k}=0$ for $i=1,2,3,4$ and $j=$ 6 or $k=6$. A system of four repetitive cells is used. However, in their studies it has not been discussed how to compensate the terms $T_{5 i j}$. Wan et al. [12] used four repetitive cells to preserve microbunching. It is based on the four cell second order achromat suggested in Refs. $[7,8]$.

From these references it is known that the $T_{5 i j}$ except $T_{566}$ for a second order achromatic bend are zero. But in an isochronous bend this element needs to be minimized as well because the residual $T_{566}$ makes a significant contribution: To preserve $0.4 \mathrm{~nm}$ microbunch with an energy spread larger than $20 \mathrm{MeV}, T_{566}$ must be less than $10^{-5} \mathrm{~m}$. This cannot be satisfied with a normal second order achromatic bend. 
In the following we describe a strategy to minimize the contribution of all $T_{5 i j}$ starting with the configuration for an achromatic bend. Equation (7) lists the expression of the $T_{5 i j}, i, j \neq 6$ elements. In addition, the terms $T_{561}, T_{562}$, and $T_{566}$ are expressed as

$$
\begin{aligned}
& T_{561}=\int_{0}^{L}\left[h\left(x \mid x_{0} \delta\right)+C_{x}^{\prime} D_{x}^{\prime}\right] d s \\
& T_{562}=\int_{0}^{L}\left[h\left(x \mid x_{0}^{\prime} \delta\right)+S_{x}^{\prime} D_{x}^{\prime}\right] d s \\
& T_{566}=\int_{0}^{L}\left[h\left(x \mid \delta^{2}\right)+\frac{1}{2} D^{\prime 2}\right] d s .
\end{aligned}
$$

$C_{x}, S_{x}, h$, and the terms such as $\left(x \mid x_{0}^{2}\right)$ are described in Eq. (7). $D$ is the dispersion and the prime ' denotes the derivative with respect to $s$. Equations (7) and (15) together list all of the nonzero terms $T_{5 i j}$.

Without losing generality we take the term $T_{511}$ as an example to demonstrate how to do the compensation. The term $\left(x \mid x_{0}^{2}\right)$ in the first of Eqs. (7) can be expressed as [8]

$$
\begin{aligned}
\left.\left(x \mid x_{0}^{2}\right)\right|_{1} \cong & -\frac{1}{2} C_{x}(s) \int_{0}^{s} K_{0}(\tau) C_{x}^{\prime 2}(\tau) S_{x}(\tau) d \tau \\
& +C_{x}(s) \int_{0}^{s} K_{2}(\tau) C_{x}^{2}(\tau) S_{x}(\tau) d \tau \\
\left.\left(x \mid x_{0}^{2}\right)\right|_{2} \cong & +\frac{1}{2} S_{x}(s) \int_{0}^{s} K_{0}(\tau) C_{x}^{\prime 2}(\tau) C_{x}(\tau) d \tau \\
& -S_{x}(s) \int_{0}^{s} K_{2}(\tau) C_{x}^{3}(\tau) d \tau .
\end{aligned}
$$

$K_{0}$ and $K_{2}$ are the dipole and sextupole strengths, respectively. There is no contribution of quadrupoles. The subscripts " 1 " and "2" refer to the solution for point to point imaging $\left(S_{x}=0\right)$ or parallel to point imaging $\left(C_{x}=0\right)$, respectively.

Following Ref. [8], the terms $C_{x}(s)$ and $S_{x}(s)$ can be expressed by two functions $\beta(s)$ and $\psi(s)$ :

$$
\begin{aligned}
C(s) & =\sqrt{\frac{\beta(s)}{\beta(0)}}[\cos \psi(s)+\alpha(0) \sin \psi(s)], \quad S(s)=\sqrt{\beta(0) \beta(s)} \sin \psi(s), \\
C^{\prime}(s) & =-\frac{(1+\alpha(0) \alpha(s)) \sin \psi(s)+(\alpha(s)-\alpha(0)) \cos \psi(s)}{\sqrt{\beta(0) \beta(s)}}, \quad S^{\prime}(s)=\sqrt{\frac{\beta(0)}{\beta(s)}}[\cos \psi(s)-\alpha(s) \sin \psi(s)],
\end{aligned}
$$

where $\alpha=-\frac{1}{2} \beta^{\prime}$. Based on these equations, the integral terms such as $\int_{0}^{s} K_{n}(\tau) C_{x}^{\prime 2}(\tau) S_{x}(\tau) d \tau$ can be expressed as

$$
F_{n}(\psi) e^{ \pm i \psi} \quad \text { and } \quad F_{n}(\psi) e^{ \pm 3 i \psi} .
$$

$F_{n}$ is a function of the magnetic field and the $\beta$ function. Substitution of Eq. (18) into Eqs. (7) yields

$$
\int_{0}^{L} h\left(x \mid x_{0}^{2}\right) d s=\sum_{k} M_{k} e^{ \pm i k \psi}, \quad k=0,2,4,
$$

and

$$
\int_{0}^{L} C_{x}^{\prime 2}(s) d s=\sum_{k} M_{k} e^{ \pm i k \psi}, \quad k=0,2,
$$

and the final result is given by

$$
T_{511}=\sum_{k} M_{k} e^{ \pm i k \psi}, \quad k=0,2,4 .
$$

Here $M_{k}$ is a function of the magnetic field and $\beta$. It is determined by the configuration of each cell.

Using Eqs. (7) and (15), the other terms $T_{5 i j}$ are obtained in an analogous fashion and can be expressed in the form

$$
T_{5 i j}=\sum_{k} M_{k} e^{ \pm i k \psi}, \quad k=0,1,2,3,4,5,6 .
$$

Now consider using repetitive cells to cancel these terms. The sum-up term $M_{k} e^{ \pm i k \psi}$ in Eq. (22) can be deemed as a complex vector: On the complex plane $\psi$ is the angle and $M_{k}$ is the vector's length. Each complex vector represents a cell. We assume there are $N$ repetitive identical cells and their total phase advance is $2 \pi$. The $M_{k}$ for each cell are identical, but the $\psi$ differ. The vector of the system is obtained by summation over $N$ cells:

$$
\begin{aligned}
M_{k} \sum_{m=1}^{N} e^{i[(2 \pi / N) k \cdot m]}= & M_{k} \frac{e^{i(2 \pi / N) k}\left(1-e^{i 2 \pi k}\right)}{1-e^{i(2 \pi / N) k},} \\
& \left(1-e^{i(2 \pi / N) k} \neq 0\right) .
\end{aligned}
$$

Since $k$ is an integer the term $1-e^{i 2 \pi k}=0$, which makes the complex vector for the whole $N$ cell system zero. This means that all of the second order terms $T_{5 i j}$ are mutually canceled. The precondition is that $1-e^{i(2 \pi / N) k} \neq 0$, so $N$ cannot be equal to $k$ and $k \neq 0$.

The phase advance over the $N$ cells is $2 \pi$, so the system's transport matrix is unity.

Making a bend second order achromatic requires sextupoles and a certain amount of dispersion. However, from Eq. (15) it is seen that a large dispersion will induce a large $T_{566}$. Consequently, a conflict is apparent: To tune a second order achromatic bend dispersion is needed which induces at the same time an unavoidable $T_{566}$. These arguments lead to the proposal to slightly violate the second order achromatic bend conditions in order to achieve a small enough $T_{566}$.

In addition, increasing the number of repetitive cells to more than four has some benefits: From Eq. (22), the 


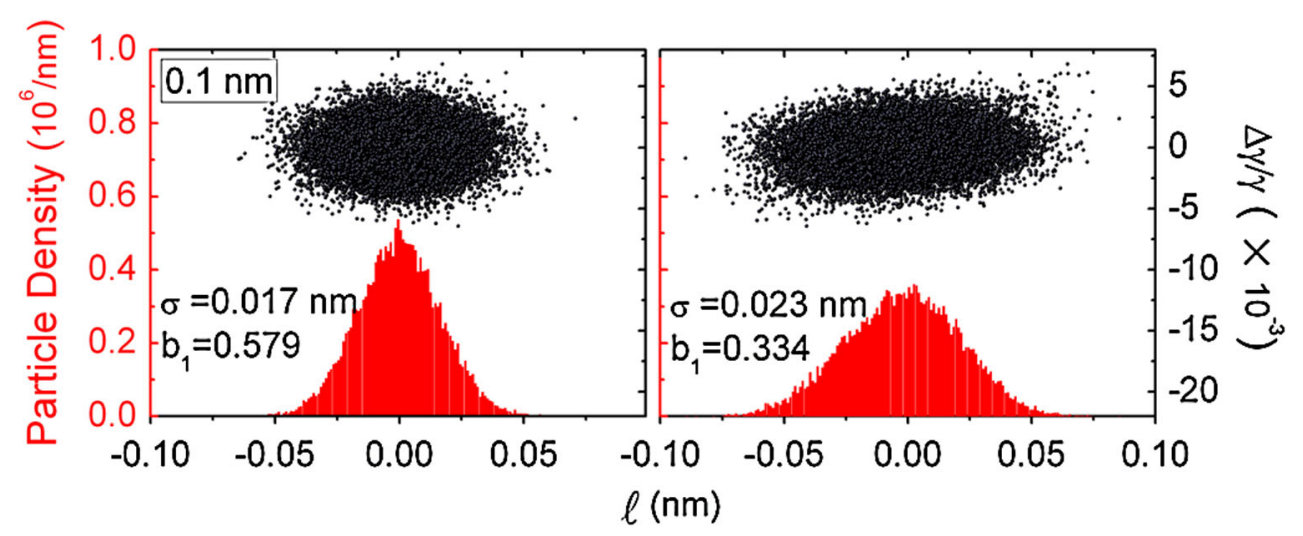

FIG. 10. (Color) Microbunch before (left) and after (right) a bend comprised by eight repetitive cells. The initial microbunch is assumed to be Gaussian with full length $(6 \sigma)$ of $0.1 \mathrm{~nm}$. 20000 sample electrons have been tracked using the code ELEGANT.

second order terms $T_{5 i j}$ include $k$ from 0 to 6 . Based on Eq. (23), at least seven identical cells are needed to compensate the terms of $k=1, \ldots, 6$. Moreover, the whole repetitive system should also be first order isochronous. Therefore conveniently an eight cell system instead of seven should be optimized.

The potential of the method described above is given in the example below. A microbunch with $0.1 \mathrm{~nm}$ full length $(6 \sigma)$ is transported through an eight cell system consisting of 72 magnets. The result is visualized in Fig. 10 in a similar fashion as Figs. 4 and 7. Note the different scales. It is seen that the bunch size is increased by about $35 \%$ and the bunch factor is decreased correspondingly by about $40 \%$. On this scale the system can preserve microbunching quite well, but not perfectly. The observed bunch expansion is attributed to residual second order terms at $k=0$. Beside its complexity of using many magnets, it needs quite strong sextupoles of $k_{2} \approx 300 \mathrm{~m}^{-3}$. The microbunch length of this example, however, is much shorter than needed for SASE3.

\section{B. Four cell bending system (FCBS) for SASE3}

For the SASE3 undulator system only $\lambda_{R}=0.1 \mathrm{~nm}$ is needed. The requirements of the previous section are too high and may be relaxed. In order to reduce the effort a four cell bending system (FCBS) was selected as a basis, which uses a configuration similar to the one proposed for the post-linac collimation section of the European XFEL $[13,14]$. Again, in order to minimize the $T_{566}$ to meet requirements, the achromat conditions are slightly violated.

The cell structure is shown schematically in Fig. 11. Two kinds of cells are used: "forward" cells " $F$ " and "reverse" cells "R." Both " $F$ " and "R" cells are comprised of two dipoles, three quadrupoles, and four sextupoles. " $F$ " and " $R$ " cells are mirror symmetric with respect to the common quadrupole. The whole FCBS is arranged as "F-R-FR." 33 magnets are used in total, including 8 dipoles, 9 quadrupoles, and 16 sextupoles. This is a significantly reduced effort as compared to the previous section.

Similar to the eight cell system the symmetry of the FCBS cannot compensate second order aberrations, but sextupoles can be used effectively to minimize them. These are the main sources of errors to be further investigated. The proposed FCBS requires about $87 \mathrm{~m}$ of total length, which is compatible with available space in the SASE3 tunnel.

The transport capabilities of the FCBS are demonstrated by Fig. 12. A $0.4 \mathrm{~nm}$ microbunch $(6 \sigma)$ is transported through the FCBS. The representation in Fig. 12 is analogous to Figs. 4 and 7. Comparing Fig. 12 with Figs. 4 and 7 there is a striking difference: After passage through the FCBS the $0.4 \mathrm{~nm}$ microbunch is only moderately expanded. The moon shaped distortion after the FCBS is due to a residual nonzero $T_{566}$.

For Fig. 12 the $R_{56}$ of the FCBS was set to zero to achieve shortest microbunch length at the exit of the FCBS. However, the $R_{56}$ can be adjusted to positive values by readjusting the dipole strengths of the FCBS. So debunching in the undulator section can be reduced as discussed in the context of Eq. (14) and Fig. 9.

The beam optical functions for the FCBS are shown in Fig. 13.

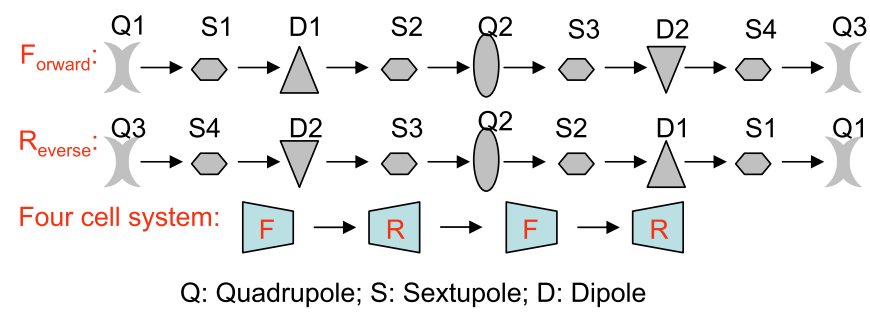

FIG. 11. (Color) Layout of the four cell bending system (FCBS). In total 33 magnets ( 8 dipoles, 9 quadrupoles, and 16 sextupoles) are used. 


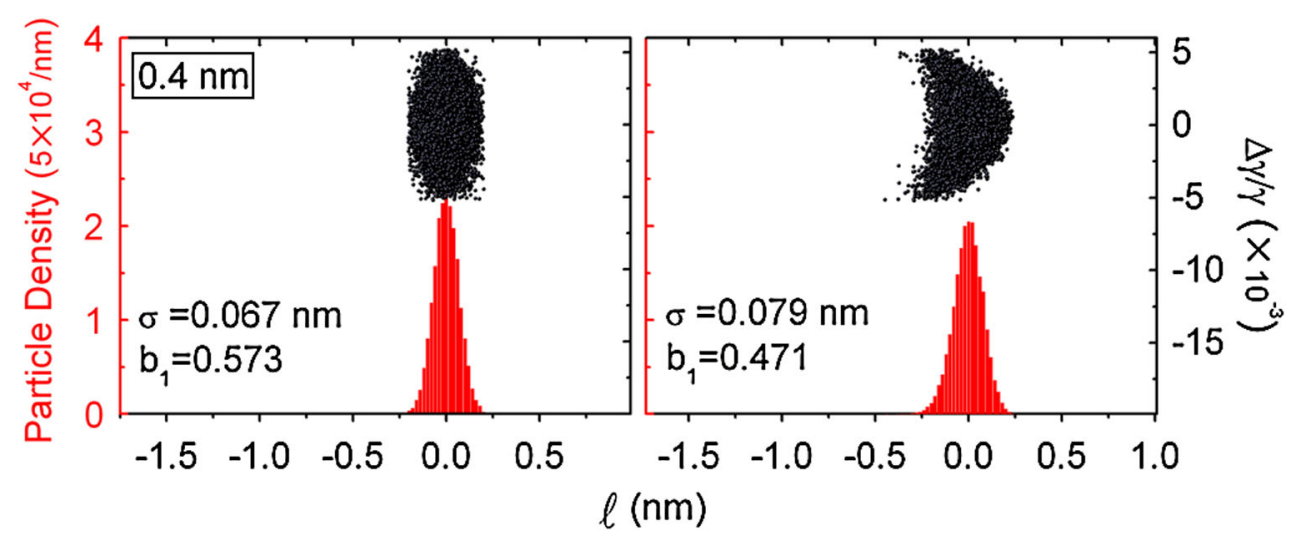

FIG. 12. (Color) Bunch expansion by the FCBS. A $0.4 \mathrm{~nm}(6 \sigma)$ Gaussian bunch before (left) and after (right) the FCBS is shown. As can be seen by the $\sigma$ values, there is only moderate expansion. The moon shaped distortion originates from a residual nonzero $T_{566}$.

\section{RADIATION PROPERTIES OF A TEST RADIATOR}

In the previous sections the capability for preserving microbunching was analyzed by transporting a Gaussian shaped bunch through the bending systems under investigation. The lengths of the bunches were chosen such that $6 \sigma$ corresponded to the radiation wavelength of interest. Only a qualitative evaluation based on relative changes of $\sigma$ or $b_{1}$ was possible. In this section a quantitative analysis is made: FEL simulations using GENESIS 1.3 [9] are used to obtain the realistic microbunching after the planar SASE3 undulator right before the bend. The microbunched electron beam is then transported through the bending system under investigation using MAD [10]. After the bend the radiation properties are calculated using GENESIS 1.3 again. These investigations are made as a function of the radiation wavelength. In this way the impact of debunching on the radiation properties of the radiator is studied.

Figure 14 shows the changes of the normalized bunch factor with respect to its initial value directly after the bend

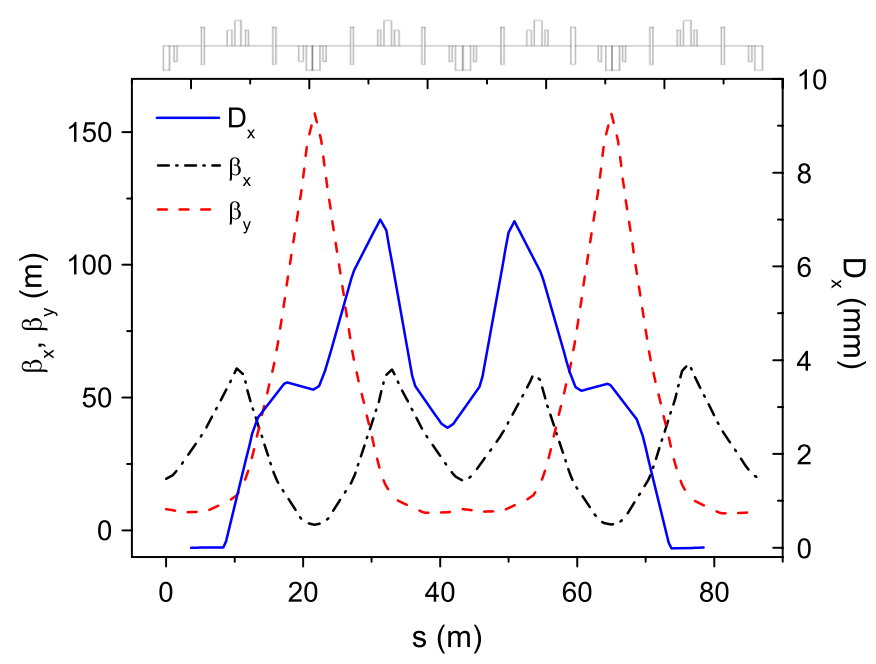

FIG. 13. (Color) Beam optical functions for FCBS. as a function of the radiation wavelength for the different kinds of bending systems treated in this paper. The bunching in the FCBS is significantly higher than for the first order systems. At short wavelengths this is most evident. The FCBS with $R_{56}=0$ shows the smallest degradation. A positive $R_{56}$ in the FCBS, however, degrades the debunching directly after the FCBS but has advantages in the undulator behind as will be shown below. This also holds for the achromatic first order system with positive $R_{56}$, the detuned first order isochronous system. Results are in qualitative agreement with the results of Secs. II and III.

In principle, any type of undulator can be used as a radiator. For the generation of circularly polarized light two different concepts might be applied. A magnetic structure producing a helical field such as an APPLE II type undulator is the most direct way. An alternative is a crossed undulator system, consisting of two planar undulators, one in the horizontal and one in the vertical plane installed in

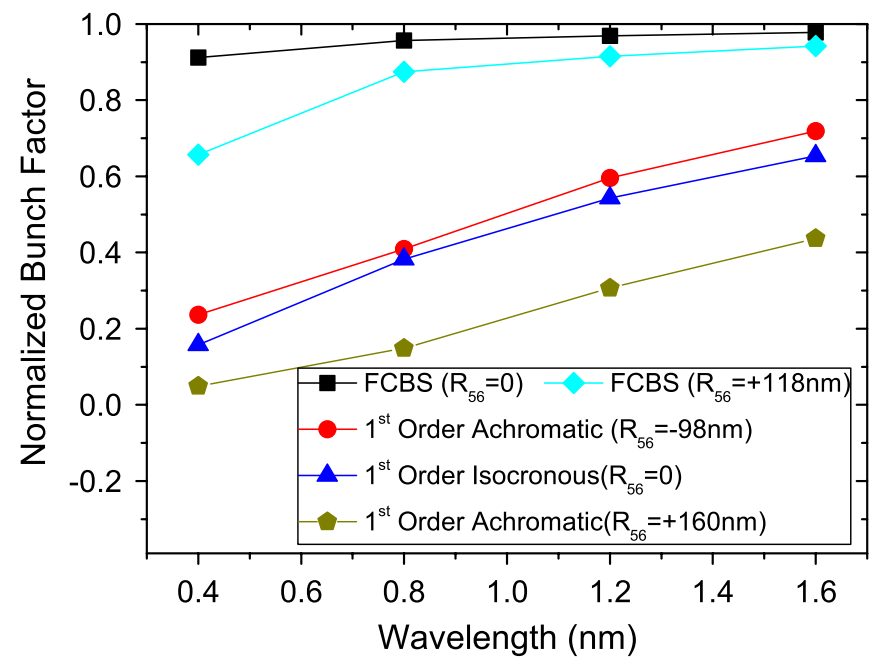

FIG. 14. (Color) Changes of the normalized bunch factor with respect to its initial value directly after the bend as a function of the radiation wavelength for the different kinds of bending systems treated in this paper. 
series. The optimum choice of technology is not the subject of this paper since each alternative has pros and cons. For this investigation a crossed undulator system [4] was selected to evaluate radiation properties for the following more practical reasons: First, for a crossed undulator system the polarization properties can be calculated with existing codes. This is done by linear superposition of the two components emitted by the two undulators with the proper phase relationship taken into account. This method is described in more detail in Ref. [15]. In contrast available codes such as GENESIS 1.3 do not permit the calculation of circular polarization properties from a helical field. Second, a crossed undulator is more sensitive to even small differences in phase space properties at the location of the two undulators and therefore is a more sensitive test device than a helical undulator, where both radiation components are generated at the same longitudinal location.

Figure 15 shows the total radiation power generated by a crossed undulator consisting of two $3 \mathrm{~m}$ long planar segments with a $90^{\circ}$ phase lag. All the different bending systems treated in this paper have been simulated. In addition and for reference, the green stars indicate the ideal case where the electron distribution after the SASE3 undulator was directly input into the radiator. Since the FCBS's preserve microbunching better than the first order systems, see Fig. 14, the radiation power for the FCBS's is significantly higher. This difference is most pronounced at short wavelengths. In addition, a positive $R_{56}$ improves bunching in the undulator and correspondingly leads to higher radiation power, which even exceeds slightly the ideal case.

Figure 16 shows the corresponding degree of circular polarization, $P_{c}$, generated by these systems. $P_{c}$ is calcu-

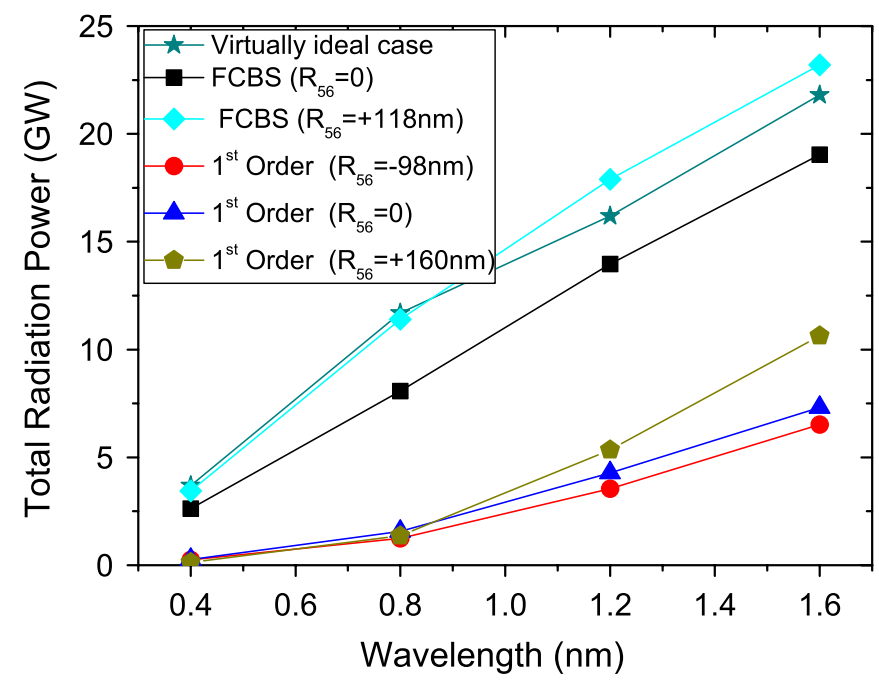

FIG. 15. (Color) Total power of a crossed planar undulator system using two $3 \mathrm{~m}$ long planar segments with a $90^{\circ}$ phase lag installed after the different kinds of bending systems discussed in this paper.

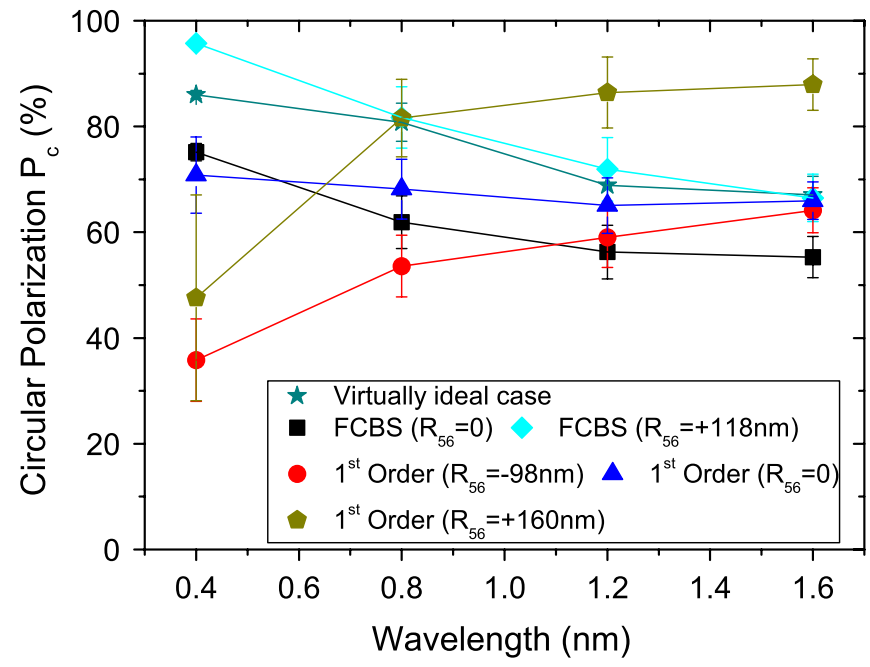

FIG. 16. (Color) Degree of circular polarization of a crossed planar undulator system using two $3 \mathrm{~m}$ long planar segments with a $90^{\circ}$ phase lag installed after the different kinds of bending systems discussed in this paper. The error bars are given by the limited simulation accuracy.

lated from the Stokes parameters using $P_{c}=S_{3} / S_{0}$. In general it is wavelength dependent.

At $0.4 \mathrm{~nm}$, only the second order systems are capable to produce $P_{c}>70 \%$. In addition, by choosing a positive $R_{56}$, it is possible to obtain polarization rates close to $100 \%$, which is again higher than the virtually ideal case. However, in Fig. 16 the $R_{56}$ value of $+118 \mathrm{~nm}$ is optimized for $\lambda_{r}=0.4 \mathrm{~nm}$ only. At longer wavelengths retuning would be required leading to an increased $P_{c}$ again.

Most first order systems show $P_{c}>\approx 50 \%$ only above $\approx 1.0 \mathrm{~nm}$. Although the curve of the first order isochronous bend as shown by the triangles in Fig. 16 has an approximately constant $P_{c}$ of $70 \%$, a comparison with Fig. 15 shows that the intensity gets very low. Above $1.0 \mathrm{~nm}$ the best results are obtained for the detuned isochronous bend by choosing a positive $R_{56}$ of $+160 \mathrm{~nm}$ as shown by the pentagons in Fig. 16.

\section{SUMMARY AND OUTLOOK}

In this paper bending systems were investigated, which preserve the microbunching obtained in a SASE FEL and simultaneously deflect the electron beam. Such a bending system could be used after the planar SASE3 undulator system of the European XFEL. In this way two beam lines can be served with FEL radiation, one with the linear polarized light of the planar SASE3 system and one with the light of a radiator installed in the deflected beam. For the European XFEL a helical radiator is of great interest. For the bending systems different solutions have been developed, which approximately preserve microbunching. Their complexities increase significantly with decreasing wavelength. Simple first order systems requiring only 3-4 dipole magnets and 1-2 quadrupoles can be used for wave- 
lengths larger than about $1 \mathrm{~nm}$. In order to cover the full wavelength range of SASE3 from 0.4 to $1.6 \mathrm{~nm}$, a significantly higher effort is required. Using a system of repetitive cells, second order corrections can to be made. The proposed four cell bending system (FCBS) requires 8 dipoles, 9 quadrupoles, 16 sextupoles, and a total length of about $87 \mathrm{~m}$. An eight cell bending system is shown to be the ultimate limit. It is capable to preserve microbunching down to $0.1 \mathrm{~nm}$ but requires more than twice the magnets than the FCBS. The first and second order isochronous systems allow in addition for the adjustment of a positive $R_{56}$, which can be used as a kind of feed forward for the negative contribution in the undulator. It increases the radiation power via an increased bunching. A crossed undulator system with a length of $2 \times 3 \mathrm{~m}$ was used to benchmark the polarization properties obtained with the different bending systems. The degree of polarization, $P_{c}$, could be $70 \%$ to almost $100 \%$ over the whole wavelength range. For the second order systems the radiation power is the range of 5-20 GW depending on wavelength. However, this paper is not an evaluation of a specific radiator system. The results show clearly that a second order bending system is superior and provides higher radiation power and better polarization properties than first order systems. A positive $R_{56}$ will further improve these properties. Above $1 \mathrm{~nm}$, however, a first order system, again with a positive $R_{56}$, might be suited with the total power reduced by a factor 2-3 as compared to the second order system.

It should be emphasized that this work is only a first step and a basic study. In this wavelength range no examples were found in the literature. So the focus was on theoretical work to find solutions and limits for a bending system suitable to preserve microbunching after the SASE3 undulator of the European XFEL. It is not a feasibility nor a design study for such a bending system. Nevertheless, first estimates on critical issues have already been made and are briefly mentioned here as an outlook to further work: coherent synchrotron radiation (CSR) has been studied qualitatively. In a bend CSR can only play a role at the entrance and exit, where the wave fronts are parallel to the orientation of the microbunches (see also Fig. 1). The influence of CSR from these short sections is considered weak and will not deteriorate beam quality. Incoherent synchrotron radiation (ISR) effects have been calculated. They play only a minor role due to the weak bending magnets. ISR induces a negligible effect on the energy spread and a small increase in emittance of $1 \%$ to $4 \%$, which is considered negligible too. Magnet strength errors have been estimated and found tolerable if they are smaller than $0.1 \%$. There are, however, strong sextupoles needed, which need more work. Finally, it should be mentioned that alignment tolerances will provide a real challenge. First ELEGANT [11] results indicate that alignment tolerances for some magnets in some of the systems discussed in this paper will be in the order of $10-50 \mu \mathrm{m}$ and might be out of reach for conventional surveying methods. In this case beam based alignment (BBA) techniques can be used. BBA is already used in large SASE FEL's such as the Linac Coherent Light Source at SLAC to align the electron beam inside the undulator, routinely with an accuracy of $2 \mu \mathrm{m}$ per gain length $(7 \mathrm{~m})$ [16]. These examples point towards possible solutions but also underline that much more design work is needed to obtain a feasible system.

\section{ACKNOWLEDGMENTS}

We thank Vladimir Balandin, Nina Golubeva, and Martin Dohlus from DESY and Alexander N. Matveenko from HZB/BESSY for many useful discussions.

[1] M. Altarelli et al., The European X-Ray Free-Electron Laser Technical Design Report, ISBN 3-935702-17-5

[2] H. Kondratenko and E. L. Saldin, Part. Accel. 10, 207 (1980).

[3] R. Bonifacio, C. Pellegrini, and L.M. Narducci, Opt. Commun. 50, 373 (1984).

[4] K. J. Kim, Nucl. Instrum. Methods Phys. Res., Sect. A 219, 425 (1984).

[5] K. J. Kim, Nucl. Instrum. Methods Phys. Res., Sect. A 445, 329 (2000).

[6] Y. Ding and Z. Huang, Phys. Rev. ST Accel. Beams 11, 030702 (2008).

[7] K. L. Brown, Report No. SLAC-75.

[8] K. L. Brown and Roger V. Servranckx, Report No. SLACPUB-3381.

[9] S. Reiche, Nucl. Instrum. Methods Phys. Res., Sect. A 429, 243 (1999).

[10] H. Grote and F.C. Iselin, Report No. CERN/SL/90-13 (AP), Rev. 5.

[11] M. Borland, User's manual for ELEGANT, available on-line at http://www.aps.anl.gov/Accelerator_Systems_Division/ Operations_Analysis/manuals/elegant_latest/elegant.html.

[12] W. Wan, J. Corlett, W. Fawley, and A. Zholents, in Proceedings of the 9th European Particle Accelerator Conference, Lucerne, 2004 (EPS-AG, Lucerne, 2004).

[13] V. Balandin, R. Brinkmann, W. Decking, and N. Golubeva, TESLA-FEL Report No. 2007-05.

[14] V. Balandin, R. Brinkmann, W. Decking, and N. Golubeva, in Proceedings of the 23rd Particle Accelerator Conference, Vancouver, Canada, 2009 (IEEE, Piscataway, NJ, 2009), TH6PFP030.

[15] Y. Li, B. Faatz, and J. Pflueger, Nucl. Instrum. Methods Phys. Res., Sect. A 613, 163 (2010).

[16] H.-D. Nuhn et al., Proceedings of the FEL2009 Conference, Liverpool, UK, 2009 (JACoW, Geneva, 2010), THOA02. 